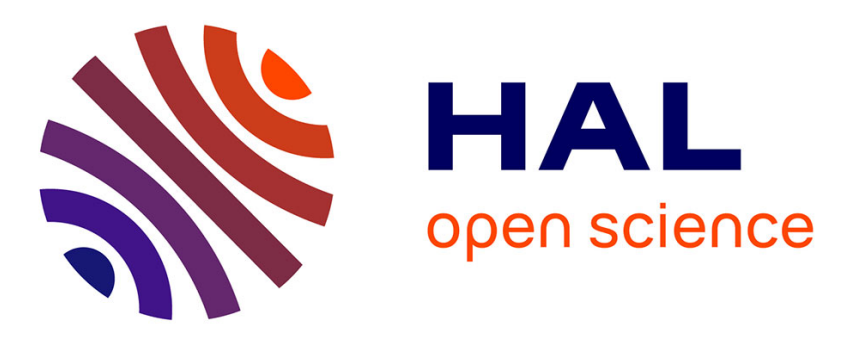

\title{
Shoulder and elbow kinematics during the Mallet score in obstetrical brachial plexus palsy
}

Olivier Herisson, Nathalie Maurel, Amadou Diop, Morgane Le Chatelier, Adeline Cambon-Binder, Franck Fitoussi

\section{- To cite this version:}

Olivier Herisson, Nathalie Maurel, Amadou Diop, Morgane Le Chatelier, Adeline Cambon-Binder, et al.. Shoulder and elbow kinematics during the Mallet score in obstetrical brachial plexus palsy. Clinical Biomechanics, 2017, 10.1016/j.clinbiomech.2017.01.006 . hal-01446112

\section{HAL Id: hal-01446112 \\ https: / hal.sorbonne-universite.fr/hal-01446112}

Submitted on 25 Jan 2017

HAL is a multi-disciplinary open access archive for the deposit and dissemination of scientific research documents, whether they are published or not. The documents may come from teaching and research institutions in France or abroad, or from public or private research centers.
L'archive ouverte pluridisciplinaire $\mathbf{H A L}$, est destinée au dépôt et à la diffusion de documents scientifiques de niveau recherche, publiés ou non, émanant des établissements d'enseignement et de recherche français ou étrangers, des laboratoires publics ou privés. 
Title: Shoulder and elbow kinematics during the Mallet score in Obstetrical brachial plexus palsy.

\section{Author names and affiliations:}

Olivier Herisson ${ }^{\mathrm{a}}$, Nathalie Maurel ${ }^{\mathrm{c}}$, Amadou Diop ${ }^{\mathrm{c}}$, Morgane Le Chatelier ${ }^{\mathrm{a}}$, Adeline Cambon-Binder ${ }^{\mathrm{b}}$, Franck Fitoussia ${ }^{\mathrm{a}}$.

a) Hopital Trousseau, Service de chirurgie orthopédique pédiatrique, 26 Avenue du Dr Arnold Netter, 75012 Paris, France.

b) Université Pierre et Marie Curie, 91 Boulevard de l'Hôpital, 75013 Paris, France.

c) Ecole Nationale Supérieure d'Arts et Métiers - Equipe Biomécanique et Remodelage Osseux, 151 boulevard de l'Hôpital, 75013 Paris, France.

Olivier Herisson : olivierherisson@hotmail.com

Nathalie Maurel : nathalie.maurel@ensam.eu

Amadou Diop : amadou.diop@ensam.eu

Morgane Lechatelier : m.lechatelier@gmail.com

Adeline Cambon-Binder : adeline_cambon@yahoo.fr

Franck Fitoussi : franck.fitoussi@aphp.fr

Corresponding author: Olivier Herisson (olivierherisson@hotmail.com)

Word count : Main text : 2549, abstract : 270

Conflicts of interest : None.

\section{$\underline{\text { Abstract: }}$}

Background: The physical signs of obstetrical brachial plexus palsy range from temporary upper-limb dysfunction to a lifelong impairment and deformity in one arm. The aim of this study was to analyze the kinematics of the upper limb and to evaluate the contribution of glenohumeral and scapulothoracic joints of obstetrical brachial plexus palsy children.

Methods: Six children participated in this study: 2 males and 4 females with a mean age of 11.7 years. Three patients had a C5, C6 lesion and 3 had a C5, C6, C7 lesion. They were asked to perform the five tasks based on the Mallet scale and the kinematic data were collected using the Fastrak electromagnetic tracking device. 
Findings: The scapulothoracic protraction and posterior tilt were significantly increased in the involved limb during the tasks Hand to mouth ( $p=0.006$ and $p=0.015$ respectively). he scapulothoracic Protraction/glenohumeral Elevation ratio was significantly increased in the involved limb during the hand to neck task $(p=0.041)$ and the elevation task $(p=0.015)$. The ratios of scapulothoracic Tilt on the three glenohumeral excursion angles were significantly increased during the hand to mouth task $(\mathrm{p} \leq 0.041)$. The scapulothoracic Mediolateral/glenohumeral Elevation ratio was significantly increased in the involved limb during the elevation task $(p=0.038)$. The glenohumeral elevation excursion was significantly decreased in the involved limb during the hand to neck task $(p<0.001)$ and the elevation task $(p=0.0003)$.

Interpretation: This study gives us information about the greater contribution of the scapulothoracic joint to shoulder motion for affected arm of obstetrical brachial plexus palsy patients compared to their unaffected arm. Kinematic analysis could be useful in shoulder motion evaluation during the Mallet score and to evaluate outcomes after surgery.

Key words: Obstetrical brachial plexus palsy, electromagnetic, kinematics, upper limb palsy, scapulothoracic joint, glenohumeral joint, reliability test.

\section{Introduction:}

Obstetrical brachial plexus palsy (OBPP) occurs at a frequency of 1 to 4 per 1000 live births $(1,2)$. OBPP produces a spectrum of injuries that vary in the severity of the nerve conduction block and its location in the brachial plexus. The physical signs of OBPP range from temporary upper-limb dysfunction to a lifelong impairment and deformity in one arm. When recovery is incomplete, patients often present a limitation of shoulder elevation and external rotation, that sometimes necessitating surgical treatment (3-7).

Clinical measures such as the Active Movement Scale and Mallet scale provide information on general strength but little on the specific joint contributions to movement $(8,9)$. Moreover, clinically significant changes in the range of movement may be detected visually by the assessor, but may not result in a change in outcome score. To quantify these changes, kinematic analysis may be a valuable adjunct to clinical tools.

Upper limbs kinematics have been widely studied in healthy, hemiplegic children or children with OBPP during functional movements (10-20). However, to our knowledge, scapulothoracic, scapulohumeral and elbow joints have never been simultaneously studied in children with OBPP. Kinematic analysis may be more informative to quantify the specific scapulothoracic joint contribution during daily living tasks.

The main objective of this study was to evaluate the shoulder kinematics (scapulothoracic and glenohumeral joints) and the elbow kinematics during the Mallet score tasks for the involved 
and non-involved limbs and to study the contribution of the glenohumeral and scapulothoracic joints. Before using a kinematic protocol in a clinical way, it is necessary to analyze its reproducibility. That is the reason why, as a secondary objective, we first quantified the within session reliability of the calculated angles for the different tasks.

\section{Methods:}

\subsection{Participants:}

Six children participated in this study: two males and four females with a mean age of 11.7 years (range, 9-14 years). Three patients had a C5, C6 lesion and three had a C5, C6, C7 lesion. Four of the children were managed conservatively whereas two underwent primary repair of the brachial plexus within the first 7 months of life (Table I).

Children were excluded from participating if they: (1) had received shoulder reconstructive surgery in the form of tendon transfers or joint releases; (2) presented with bilateral brachial plexus birth injuries; or (3) were unable to follow directions. Institutional Review Board approved the study for children. Signed assent from participants and signed consent from a parent were received prior to participation.

\subsection{Kinematic analysis:}

Four segments were included in this analysis (trunk, scapula, humerus, forearm) and three joints were considered: scapulathoracic (ST), glenohumeral (GH), elbow. Kinematic measurements were performed using the Fastrak (Polhemus, Colchester, US) electromagnetic tracking device: a portable, inexpensive movement analysis system. It included four receivers (defining technical frames) and a stylus (used for digitization purposes). The transmitter was placed just behind the scapula, the subject being in a standing position. Two receivers were fixed on rigid supports which were then linked to the forearm using straps and on the arm using an elbow-guard with two holes at the medial and lateral epicondyles to allow future palpation of these points. Two other receivers were directly fixed on skin using a doublesided tape: a first one at sternum and a second one on the flat part of the acromial process $(21,22)$ of the scapula. The fixation was reinforced by adhesive tape above the sensors.

Anatomical frames were defined for trunk, humerus and forearm as previously described $(23,24)$. For the scapula, the anatomical frame was defined using the Trigonum Spinae Scapulae (TS), the Angulus Inferior (AI), Angulus Acromialis (AA) and according to ISB recommendations (19).

The relative positions of anatomical and technical frames were defined during a static acquisition phase where anatomical points defining the anatomical frames were digitized using the stylus, the receivers being simultaneously linked to the segments.

Angular kinematic calculations were then performed using anatomical frames and sequences as described previously (24), except for the scapulothoracic and scapulohumeral motions which were based on Brochard et al (25). Finally, eight angular motions were studied: the Protraction/retraction (STPro), the Medial/lateral rotation (STMed) and the Anterior/posterior 
tilt (STTilt) angular motions for the scapulothoracic joint, the Plane of elevation (GHPE), the Elevation (GHEle) and the Internal/External Rotation (GHRot) angular motions for the glenohumeral joint and the flexion/ extension and pronation/supination for the elbow. An illustration of the 3D motions can be found in Brochard et al. (25)

\subsection{Procedure:}

Subjects stood with their feet at a comfortable width apart and the posterior aspect of the heel in contact with a thin L-shaped valley fixed on the floor. Subjects were asked to look forward while moving. To mobilize the upper limb in different directions in space, the patients were asked to perform five tasks based on the Mallet scale (Figure I):

First task: Hand to mouth.

Second task: Hand to neck.

Third task: Hand to back.

Fourth task: elevating the upper limb in the scapular plane. During arm elevation the elbow was extended. The scapular plane was defined at $40^{\circ}$ from the frontal plane and going through the acromion. To standardize the movement, a laser spot was fixed at the external part of the arm and the subject was asked to follow with the laser spot a vertical line that intersects the scapular plane and a plane perpendicular to the scapular plane.

For these four previous tasks, at the initial position, the upper limbs were placed in the anatomical reference position.

Fifth task: external rotation. The elbow was flexed at $90^{\circ}$ with the forearm in a sagittal plane at initial position.

This assessment was standardized: Orthopedic Surgeon $(\mathrm{OH})$ was responsible for sensor placement. The whole procedure was successively carried out for the involved and intact sides. For a given arm, each task was repeated twice consecutively to assess the within session reproducibility. The mallet scale assessment was done during the same procedure. This evaluation was done by the same orthopedic surgeon $(\mathrm{OH})$.

\subsection{Data analysis:}

The angular excursions were calculated between the initial and the final positions. For the elevation task, the final position was either $90^{\circ}$ of the humeral elevation if possible or the maximum value if below. Movement cycles were time-normalized (0-100\%) and each joint angle can be visualized as a function of cycle percentage. The angular value at the initial position was also analyzed. We finally calculated the ratios between ST joint excursion angles and $\mathrm{GH}$ joint excursion angles.

\subsection{Statistical analysis:}

Discriminative ability. 
We compared parameters between the involved limb group and the intact limb group. We first analyzed if the normality assumption could be accepted using Lilliefors test for the two compared groups (the mean of the two repetitions for each group). When normality existed for the two groups, we used an independent sample t-test with the assumption of unequal variance. Otherwise, we used a Wilcoxon signed rank test. Statistical significance was set at $p$ $\leq 0.05$.

\subsection{Reliability:}

The within session reliability of parameters was analyzed using the intraclass correlation coefficient (ICC) with an ICC $(2,1)$ model (26). The reliability was classified as excellent (ICC $\geq 0.90$ ), very good ( $(\mathrm{ICC} \geq 0.80$ ), good (ICC $\geq 0.70$ ), moderate (ICC $\geq 0.60$ ) or poor otherwise.

\section{Results:}

Results concerning the clinical Mallet score assessments are depicted in Table II. An example of the normalized kinematic curves is given in Figure II. Angular excursions for non-involved and involved limbs are presented in Table IV.

\subsection{Reliability: (Table III)}

For the involved limb, the reliability of ST and GH angular excursions was good to excellent for all the tasks.

For the non-involved limb, the reliability of ST angular excursions was very good to excellent for all the tasks except STPro during the hand to back $(\mathrm{ICC}=0.5)$ and the external rotation tasks $(\mathrm{ICC}=0.6)$ and STTilt during the elevation task $(\mathrm{ICC}=0.6)$. The reliability of $\mathrm{GH}$ angular excursions was very good to excellent for all the tasks except the GHRot during the hand to neck task ( $\mathrm{ICC}=0.3$ ) and the GHEle during the external rotation task $(\mathrm{ICC}=0.1)$.

The reliability was good to excellent for all the initial angular position, for all the tasks and for the non-involved and involved limbs.

\subsection{Kinematic analysis:}

This analysis includes only data having an ICC higher or equal to 0.6 for the involved and the non-involved limbs which are considered to be the most relevant.

\subsubsection{Initial Position (Figure III-A).}

The scapula was oriented more medially for the involved limb $\left(20.4^{\circ}\right)$ than for the intact limb $\left(6.5^{\circ}\right)$ and it was significant for all the tasks $(p<0.022)$ except for the hand to back task $(\mathrm{p}=0.053))$

The elbow of the involved limb was significantly more flexed $\left(39.3^{\circ}\right.$ vs $\left.18.1^{\circ}\right)(\mathrm{p}<0.034)$ for all the tasks except for the external rotation task.

The GH joint had a significant greater elevation for the involved limb $\left(-12.1^{\circ}\right)$ than for the intact limb $\left(2.4^{\circ}\right)(\mathrm{p}<0.025)$ except for the hand to back task $(\mathrm{p}=0.086)$. 


\subsubsection{Hand to mouth.}

ST protraction and posterior tilt were significantly increased in the involved limb $(p=0.006$ and $\mathrm{p}=0.015$ respectively). Elbow flexion was significantly decreased in the involved limb $(\mathrm{p}=0.007)$. Concerning the contribution of $\mathrm{ST}$ and $\mathrm{GH}$, the three ratios STTilt/GHPE, STTilt/GHR, STTilt/GHEle were significantly increased in the involved limb $(p=0.026$, $\mathrm{p}=0.005$ and $\mathrm{p}=0.041$ respectively). The STPro/GHPE was also significantly increased in the involved limb $(p=0.041)$. Figure III-B illustrates the 3D positions of the scapula and of the humerus and $\mathrm{ST} / \mathrm{GH}$ ratios for this task.

\subsubsection{Hand to neck.}

There was no statistical difference between the two limbs concerning the ST and the elbow angular excursions. GH elevation excursion was significantly decreased in the involved limb $(\mathrm{p}<0.001)$. Concerning the contribution of ST and $\mathrm{GH}$, the STPro/GHEle ratio was significantly increased in the involved $\operatorname{limb}(\mathrm{p}=0.041)$.

\subsubsection{Hand to back.}

$\mathrm{GH}$ internal rotation was significantly decreased in the involved limb $(\mathrm{p}=0.002)$. The elbow flexion was significantly decreased in the involved limb $(p=0.019)$. There was no statistical difference between the two limbs concerning the ST angular excursion and the ST/GH ratios.

\subsubsection{Elevating the upper limb in the scapular plane.}

$\mathrm{GH}$ elevation was significantly decreased in the involved $\operatorname{limb}(\mathrm{p}=0.0003)$. There was no statistical difference between the two limbs concerning the ST angular excursions. The STMed/GHEle ratio was significantly increased in the involved limb $(p=0.038)$ and the STPro/GHEle was also significantly increased $(p=0.015)$. Figure III-B illustrates the 3D positions of the scapula and of the humerus and ST/GH ratios for this task. Figure IV illustrates the STMed angles during the arm elevation of the 6 patients from the initial to the final position.

\subsubsection{External rotation.}

$\mathrm{GH}$ external rotation was significantly decreased in the involved limb $(\mathrm{p}=0.004)$. Concerning the contribution of ST and $\mathrm{GH}$, there were no significant differences between the noninvolved and the involved limbs.

\section{Discussion:}

Upper limbs kinematics have been widely studied in healthy and pathologic children during functional movement (10-23) but few of them focused on children with obstetrical brachial plexus palsy $(13,14,23)$. To our knowledge, this is the first study that quantifies the 3D scapulothoracic, scapulohumeral and elbow joints mobility during the Mallet score functional 
motion in the same analysis in OBPP children. Moreover, we presented data not only about ranges of motion, but also about initial position of the different segments. We finally provides data about the within session reliability, which was not done in the previous studies.

The present kinematic protocol appears to be a useful procedure to quantify the upper limb mobility for the clinical outcome and the surgical or medical treatment planning. Upper limb kinematic analysis provides valuable, objective and quantified data to clinicians. Kinematic evaluation of children with OBPP could also help to compare the improvement of shoulder range of motion before and after treatment. It can be used as an adjunct to clinical scales such as the Mallet Scale.

Our results about angular excursions of ST and GH joints are consistent with those of previous studies. This study confirms that children with OBPP generally employ greater than average scapular motion in the involved limb during activities of daily living $(13-15,17)$ even if the differences were not always significant. Increased scapular mobility is likely a consequence of muscle imbalance, skeletal deformity, and neural adaptation. In order to analyze the compensation performed by the scapulothoracic joint in the overall shoulder range of motion, we calculated the ratio between the ST joint excursion and the GH joint excursion for the involved and the non-involved limb. The comparison of the ST/GH ratio between the two limbs evaluates the contribution of each joint in the shoulder range of motion. An increase in the ST/GH ratio reflects a greater contribution of the scapulothoracic joint or a lower contribution of the glenohumeral joint to a given movement.

Children with Erb's palsy (C5C6 and C5C6C7) also often demonstrated reduced GH motion. These modifications were responsible for the upper ST/GH ratios seen in the OBPP during some tasks of Mallet scale.

According to Duff et al., in children with limited arm elevation, the ST medial rotation in the involved limb made a larger contribution than the GH joint (13). Mosqueda et al. investigated upper limb kinematics in a group of patients with OBPP of the upper roots and showed that there were kinematic anomalies in three dimensions during various tasks performed: children with OBPP used more shoulder flexion or abduction in some activities of daily living than control subjects (14). According to Russo et al., children with OBPP demonstrated significantly greater ST motion than an unaffected group during the hand to mouth task. Moreover, the modified Mallet classification does not provide any insight into the ST or GH contributions to shoulder function. All of the patients with the same mallet scale had variable ST and GH range of motion. A large variation in ST and GH joint contributions may result in the same Mallet score (17).

For children with Erb's palsy, the dorsal scapular (Rhomboids and levator scapulae muscles), long thoracic (serratus anterior muscle) and spinal accessory nerve (Trapezius muscle) are non-involved. Normal innervation to the parascapular muscles is typically maintained $(15,20,27)$.

The greater contribution of serratus anterior and pectoralis minor could explain the increase of the ST range of motion in protraction and posterior tilt for the hand to mouth task. ST lateral 
rotation and protraction are also increased due to serratus anterior and pectoralis minor during the hand to neck task.

The contribution of serratus anterior and pectoralis minor could explain the increase of STPro/GHPE ratio in the involved limb during the hand to mouth task and of STMed/GHEle during the elevation task.

There are some limitations in this study. First, movement procedure has likely some variation that may affect the validity of kinematic measures. However movement assessment was standardized with each task that was repeated twice and guided by a surgical resident. Moreover the within session reliability study underlined a good to excellent reliability in most of the cases. Second, acromial sensor placement and soft tissues artefacts could expose to a measurement bias. The method of studying the scapula kinematics using a skin-fixed sensor was validated for adults but not for children (21). To avoid measurement errors which can occur above 90 degrees of the humeral elevation during the elevation task (28), we limited data analysis to this value, which corresponds to grade IV of Mallet classification. Last, children in this study represent a variety of nerves injuries which is a potentially confounding factor. It would be interesting to analyze a greater number of patients in each group (C5C6 or C5C6C7)

\section{Conclusions:}

We have developed a kinematic protocol for analysis of the upper limb children with obstetrical brachial plexus palsy to complete their clinical examination with objective, quantified data during the Mallet scale tasks. This study provided support for the clinical observation of inter-limb differences in shoulder and elbow movement patterns in the involved and non-involved limbs of children with brachial plexus birth palsy. Kinematic analysis may be used as an adjunct to clinical scales such as the Mallet Scale to document baseline performance along with outcomes from therapeutic and surgical intervention. Our first observations will nevertheless have to be confirmed by studying a larger number of patients.

Acknowledgement: The authors would like to thank Marine Veyrat, an engineering student who participated in this study.

\section{Conflicts of interest : None.}

\section{$\underline{\text { References: }}$}

1. Hoeksma AF, Wolf H, Oei SL. Obstetrical brachial plexus injuries: incidence, natural course and shoulder contracture. Clin Rehabil. 2000 Oct;14(5):523-6.

2. Mollberg M, Hagberg H, Bager B, Lilja H, Ladfors L. High birthweight and shoulder dystocia: the strongest risk factors for obstetrical brachial plexus palsy in a Swedish population-based study. Acta Obstet Gynecol Scand. 2005 Jul;84(7):654-9. 
3. Waters PM. Update on management of pediatric brachial plexus palsy. J Pediatr Orthop Part B. 2005 Jul;14(4):233-44.

4. Hoeksma AF, Ter Steeg AM, Dijkstra P, Nelissen RGHH, Beelen A, de Jong BA. Shoulder contracture and osseous deformity in obstetrical brachial plexus injuries. J Bone Joint Surg Am. 2003 Feb;85-A(2):316-22.

5. Waters PM. Comparison of the natural history, the outcome of microsurgical repair, and the outcome of operative reconstruction in brachial plexus birth palsy. J Bone Joint Surg Am. 1999 May;81(5):649-59.

6. Michelow BJ, Clarke HM, Curtis CG, Zuker RM, Seifu Y, Andrews DF. The natural history of obstetrical brachial plexus palsy. Plast Reconstr Surg. 1994 Apr;93(4):675-80; discussion 681.

7. Dodds SD, Wolfe SW. Perinatal brachial plexus palsy. Curr Opin Pediatr. 2000 Feb;12(1):40-7.

8. Curtis C, Stephens D, Clarke HM, Andrews D. The active movement scale: an evaluative tool for infants with obstetrical brachial plexus palsy. J Hand Surg. 2002 May;27(3):470-8.

9. Mallet J. [Obstetrical paralysis of the brachial plexus. II. Therapeutics. Treatment of sequelae. Results of different therapeutic technics and indications]. Rev Chir Orthopédique Réparatrice Appar Mot. 1972;58:Suppl 1:192-6.

10. Lempereur M, Brochard S, Mao L, Rémy-Néris O. Validity and reliability of shoulder kinematics in typically developing children and children with hemiplegic cerebral palsy. J Biomech. 2012 Jul 26;45(11):2028-34.

11. Coley B, Jolles BM, Farron A, Bourgeois A, Nussbaumer F, Pichonnaz C, et al. Outcome evaluation in shoulder surgery using 3D kinematics sensors. Gait Posture. 2007 Apr;25(4):523-32.

12. Chang J-J, Wu T-I, Wu W-L, Su F-C. Kinematical measure for spastic reaching in children with cerebral palsy. Clin Biomech Bristol Avon. 2005 May;20(4):381-8.

13. Duff SV, Dayanidhi S, Kozin SH. Asymmetrical shoulder kinematics in children with brachial plexus birth palsy. Clin Biomech Bristol Avon. 2007 Jul;22(6):630-8.

14. Mosqueda T, James MA, Petuskey K, Bagley A, Abdala E, Rab G. Kinematic assessment of the upper extremity in brachial plexus birth palsy. J Pediatr Orthop. 2004 Dec;24(6):695-9.

15. Price A, Tidwell M, Grossman JA. Improving shoulder and elbow function in children with Erb's palsy. Semin Pediatr Neurol. 2000 Mar;7(1):44-51.

16. Bialocerkowski AE, Wrigley T, Galea M. Reliability of the V-scope system in the measurement of arm movement in children with obstetric brachial plexus palsy. Dev Med Child Neurol. 2006 Nov;48(11):913-7.

17. Russo SA, Kozin SH, Zlotolow DA, Thomas KF, Hulbert RL, Mattson JM, et al. Scapulothoracic and glenohumeral contributions to motion in children with brachial plexus birth palsy. J Shoulder Elb Surg Am Shoulder Elb Surg Al. 2014 Mar;23(3):327-38.

18. Fitoussi F, Maurel N, Diop A, Laassel EM, Ilharreborde B, Presedo A, et al. Upper extremity kinematics analysis in obstetrical brachial plexus palsy. Orthop Traumatol Surg Res OTSR. 2009 Sep;95(5):336-42.

19. Wu G, van der Helm FCT, Veeger HEJD, Makhsous M, Van Roy P, Anglin C, et al. ISB recommendation on definitions of joint coordinate systems of various joints for the reporting of human joint motion--Part II: shoulder, elbow, wrist and hand. J Biomech. 2005 May;38(5):981-92.

20. Pearl ML. Shoulder problems in children with brachial plexus birth palsy: evaluation and management. J Am Acad Orthop Surg. 2009 Apr;17(4):242-54.

21. Karduna AR, McClure PW, Michener LA, Sennett B. Dynamic measurements of 
three-dimensional scapular kinematics: a validation study. J Biomech Eng. 2001 Apr;123(2):184-90.

22. Roren A, Fayad F, Poiraudeau S, Fermanian J, Revel M, Dumitrache A, et al. Specific scapular kinematic patterns to differentiate two forms of dynamic scapular winging. Clin Biomech Bristol Avon. 2013 Oct;28(8):941-7.

23. Fitoussi F, Diop A, Maurel N, Laassel EM, Penneçot GF. Kinematic analysis of the upper limb: a useful tool in children with cerebral palsy. J Pediatr Orthop Part B. 2006 Jul;15(4):247-56.

24. Maurel N, Diop A, Gouelle A, Alberti C, Husson I. Assessment of upper limb function in young Friedreich ataxia patients compared to control subjects using a new threedimensional kinematic protocol. Clin Biomech Bristol Avon. 2013 Apr;28(4):386-94.

25. Brochard S, Lempereur M, Mao L, Rémy-Néris O. The role of the scapulo-thoracic and gleno-humeral joints in upper-limb motion in children with hemiplegic cerebral palsy. Clin Biomech Bristol Avon. 2012 Aug;27(7):652-60.

26. Pecoraro F, Mazzà C, Cappozzo A, Thomas EE, Macaluso A. Reliability of the intrinsic and extrinsic patterns of level walking in older women. Gait Posture. 2007 Sep;26(3):386-92.

27. Kozin SH. The Evaluation and Treatment of Children With Brachial Plexus Birth Palsy. J Hand Surg. 2011 Aug;36(8):1360-9.

28. Lempereur M, Leboeuf F, Brochard S, Rémy-Néris O. Effects of glenohumeral joint centre mislocation on shoulder kinematics and kinetics. Comput Methods Biomech Biomed Engin. 2014;17 Suppl 1:130-1. 


\section{Legends:}

Table I: Population description.

Table II: Means and standard deviation of the Mallet scores.

Table III: Within session reliability of the scapulothoracic and glenohumeral angular excursion.

ICC (95\% CI): Intraclass correlation coefficient (95\% Confidence Interval)

Table IV: Mean and standard deviation of angular displacement between the initial and the final position. ${ }^{*} \mathrm{p} \leq 0.05 ; * * \mathrm{p} \leq 0.01 ; * * * \mathrm{p} \leq 0.001$

Figure I. View of the experimental set-up and tasks based on Mallet classification.

A: Global view; B: Elevation in the scapular plane; C: Hand to back; D: Hand to mouth; E: External rotation; F: Hand to neck

Figure II. Kinematic curves $\left(^{\circ}\right)$ during Hand to mouth task for the involved arm of a given patient. The 2 repetitions of the task and the mean value (thick line) are represented; GlenoHumeral joint (A), Scapulo-Thoracic joint (B) and elbow (C) with in abscisse the \% of the cycle.

Figure III: 3D positions of the scapula and of the humerus for the involved (in red) and the non-involved (in blue) and ST/GH ratios. A- Mean initial position for the hand to mouth, hand to neck, hand to back and elevation tasks for which the initial position was the anatomical reference position. B- Final positions for the hand to mouth and elevation tasks. For those two tasks, ST/GH ratios whose differences were significant between the involved and the non-involved limbs are presented.

Figure IV. Comparison between involved and non-involved limb: STMed angle during the Elevation task for the 6 patients from the initial to the final position. The non-involved limb in red and the involved limb in blue.

\begin{tabular}{|c|c|c|c|c|c|c|c|c|}
\hline Patient ID & DDN & Age (Years) & Șex & Involved side & Lesion & Surgery & Age when surgery & Mallet score \\
\hline & $128 / 11 / 03$ & 12 & $\mathrm{M}$ & $\mathbf{R}$ & C5C6C7 & Graft C5-TPS; C7-TPM & 6 mois & 14 \\
\hline & $231 / 03 / 03$ & 12 & $\mathrm{~F}$ & $\mathrm{~L}$ & C5C6 & & & 12 \\
\hline & $420 / 09 / 06$ & 9 & $\mathrm{~F}$ & $\mathrm{~L}$ & C5C6 & & & 17 \\
\hline & $502 / 04 / 04$ & 11 & $\mathrm{~F}$ & $R$ & C5C6 & & & 17 \\
\hline & $615 / 09 / 03$ & 12 & $M$ & $R$ & C5C6C7 & Graft C5-TPS; C6-TPS; C7-C7; spinal SS & 7 mois & 14 \\
\hline & $7 \longdiv { 2 4 / 1 2 / 0 1 }$ & 14 & $\mathrm{~F}$ & $R$ & C5C6C7 & & & 12 \\
\hline
\end{tabular}

Table I: Population description.

\begin{tabular}{lll} 
Task & Mean & SD \\
\hline Abduction & 3.17 & 1.00 \\
RECC & 2.33 & 0.50 \\
Hand to head & 2.50 & 1.26 \\
Hand to Back & 3.00 & 1.00 \\
Hand to mouth & 3.17 & 1.26
\end{tabular}


Table II: Means and standard deviation of the Mallet scores.

\begin{tabular}{|c|c|c|c|c|c|c|c|c|c|c|}
\hline \multirow{2}{*}{ ICC $(95 \% \mathrm{Cl})$} & \multicolumn{5}{|l|}{ Healthy } & \multicolumn{5}{|l|}{ Pathologic } \\
\hline & Hand to neck & Hand to Back & Elevation & External Rotation & Hand to mouth & Hand to neck & Hand to Back & Elevation & External Rotation & Hand to mouth \\
\hline $\begin{array}{l}\text { ST Med }(>0) \\
\text {-at }(<0)\end{array}$ & $0.98(0.89-1.0)$ & $0.86(0.32-0.98)$ & $0.98(0.88-1.0)$ & $0.77(0.05-0.96)$ & $0.94(0.64-0.99)$ & $0.97(0.78-1.0)$ & $0.86(0.37-0.98)$ & $0.97(0.81-1.0)$ & $0.94(0.64-0.99)$ & $0.99(0.75-1.0)$ \\
\hline $\begin{array}{l}\text { ST Tilt Ant }(<0) \\
\text { Post(>0) }\end{array}$ & $0.84(0.25-0.98)$ & $0.84(0.30-0.97)$ & $0.62(-0.23-0.94)$ & $0.94(0.68-0.99)$ & $0.79(0.07-0.97)$ & $0.98(0.87-1.0)$ & $0.85(0.28-0.98)$ & $0.96(0.80-0.99)$ & $0.86(0.35-0.98)$ & $0.98(0.88-1.0)$ \\
\hline $\begin{array}{l}\text { ST Pro }(>0) \\
\operatorname{Ret}(<0)\end{array}$ & $0.91(0.54-0.99)$ & $0.51(-0.25-0.91)$ & $0.78(0.04-0.97)$ & $0.59(-0.43-0.93)$ & $0.81(-0.07-0.97)$ & $0.81(0.22-0.97)$ & $0.94(0.66-0.99)$ & $0.88(0.36-0.98)$ & $0.73(-0.02-0.96)$ & $0.71(-0.03-0.95)$ \\
\hline $\begin{array}{l}\text { GH PE Post }(<0) \\
\text { Ant }(>0)\end{array}$ & $0.87(0.40-0.98)$ & $0.83(0.25-0.97)$ & $0.79(0.01-0.97)$ & $0.79(0.15-0.97)$ & $0.97(0.12-1.0)$ & $0.75(0.09-0.96)$ & $0.90(0.46-0.99)$ & $0.92(0.54-0.99)$ & $0.83(0.31-0.97)$ & $0.96(0.77-0.99)$ \\
\hline $\begin{array}{l}\text { GH Rot Ext(<0) } \\
\operatorname{lnt}(>0)\end{array}$ & $0.33(-0.81-0.88)$ & $0.94(0.57-0.99)$ & $0.92(0.52-0.99)$ & $0.88(0.39-0.98)$ & $0.95(0.71-0.99)$ & $0.98(0.90-1.0)$ & $0.86(0.39-0.98)$ & $0.89(0.49-0.98)$ & $0.89(0.48-0.98)$ & $0.98(0.88-1.0)$ \\
\hline $\begin{array}{l}\text { GH Ele Add }(>0) \\
\text { Abd }(<0)\end{array}$ & $0.90(0.45-0.99)$ & $0.86(0.29-0.98)$ & $0.91(0.56-0.99)$ & $0.06(-0.64-0.77)$ & $0.98(0.87-1.0)$ & $0.97(0.81-1.0)$ & $0.84(0.26-0.98)$ & $0.95(0.66-0.99)$ & $0.84(0.28-0.98)$ & $0.98(0.99-1.0)$ \\
\hline $\begin{array}{l}\text { Elbow Fle }(>0) \\
\text { Ext }(<0)\end{array}$ & $0.91(0.53-0.99)$ & $0.92(0.53-0.99)$ & $0.82(-0.07-0.98)$ & $0.72(0.02-0.96)$ & $0.98(0.82-1.0)$ & $1.0(0.99-1.0)$ & $1.0(0.99-1.0)$ & $0.74(0.03-0.96)$ & $0.1(-0.97-0.81)$ & $1.0(0.97-1.0)$ \\
\hline $\begin{array}{l}\text { Elbow Pro }(>0) \\
\text { Sup }(<0)\end{array}$ & $0.88(0.47-0.98)$ & $0.84(0.21-0.98)$ & $0.71(-0.06-0.95)$ & $0.05(-0.91-0.79)$ & $0.95(0.66-0.99)$ & $0.93(0.53-0.99)$ & $0.74(-0.1-0.96)$ & $0.94(0.67-0.99)$ & $0.32(-0.56-0.87)$ & $0.97(0.84-1.0)$ \\
\hline
\end{tabular}

Table III: Within session reliability of the scapulothoracic and glenohumeral angular excursion.

ICC (95\%CI): Intraclass correlation coefficient (95\% Confidence Interval)

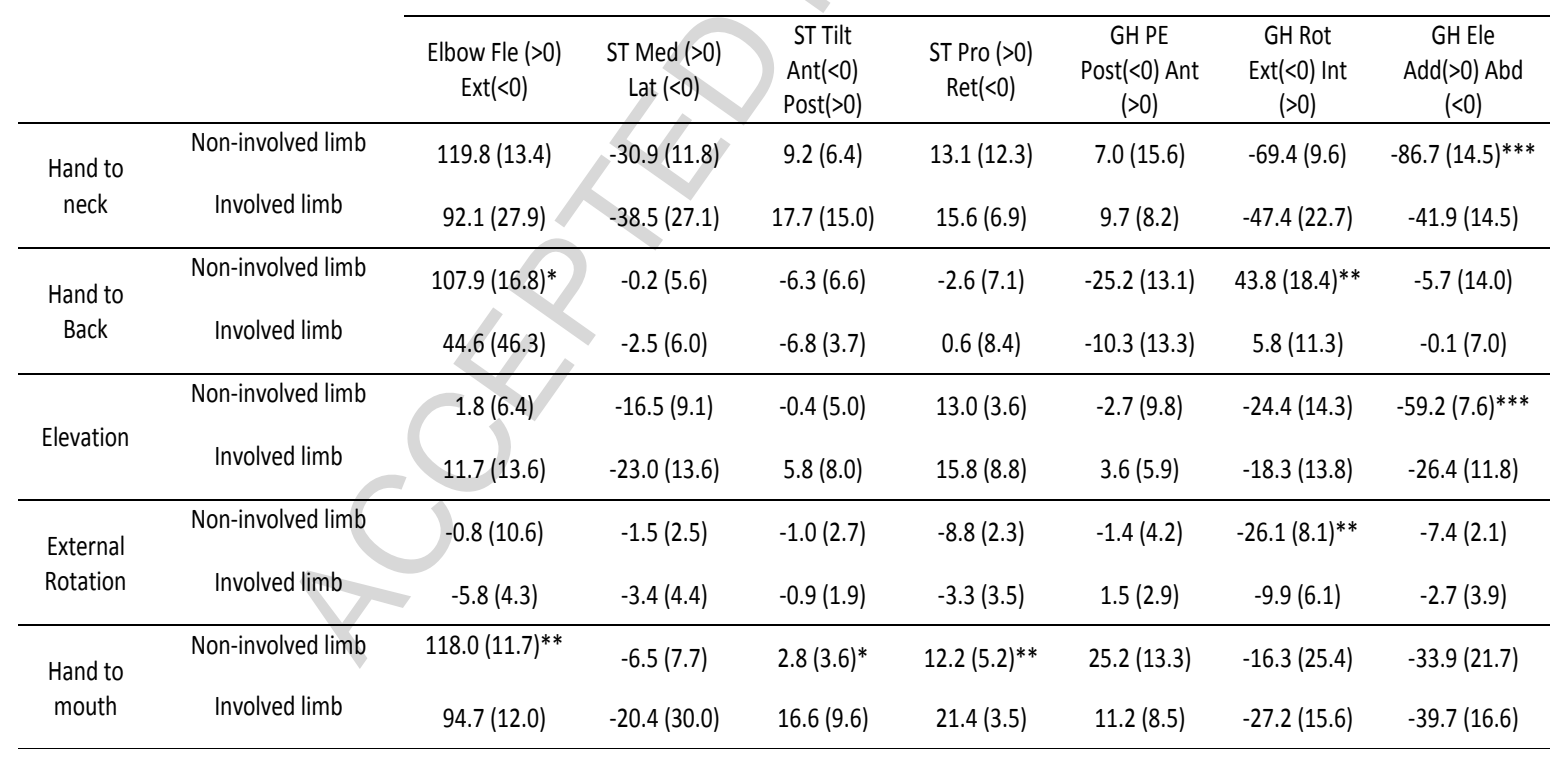

Table IV: Mean (Standard Deviation) of angular displacement between the initial and the final position. ${ }^{*} \mathrm{p} \leq 0.05 ; * * \mathrm{p} \leq 0.01 ; * * * \mathrm{p} \leq 0.001$ 


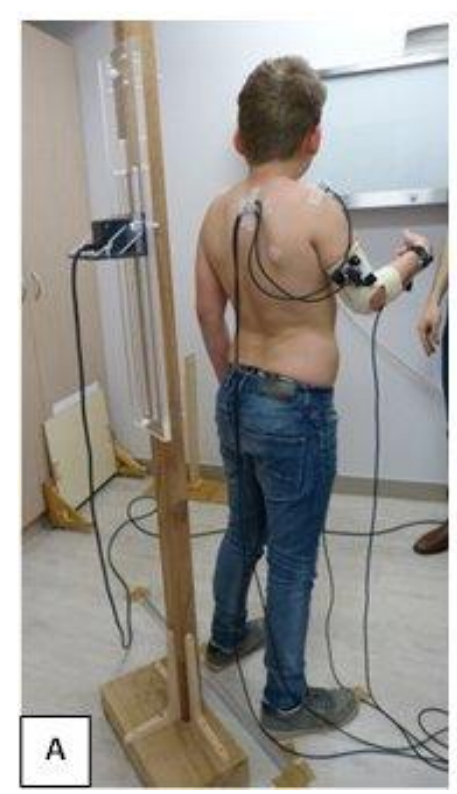

Fig. 1

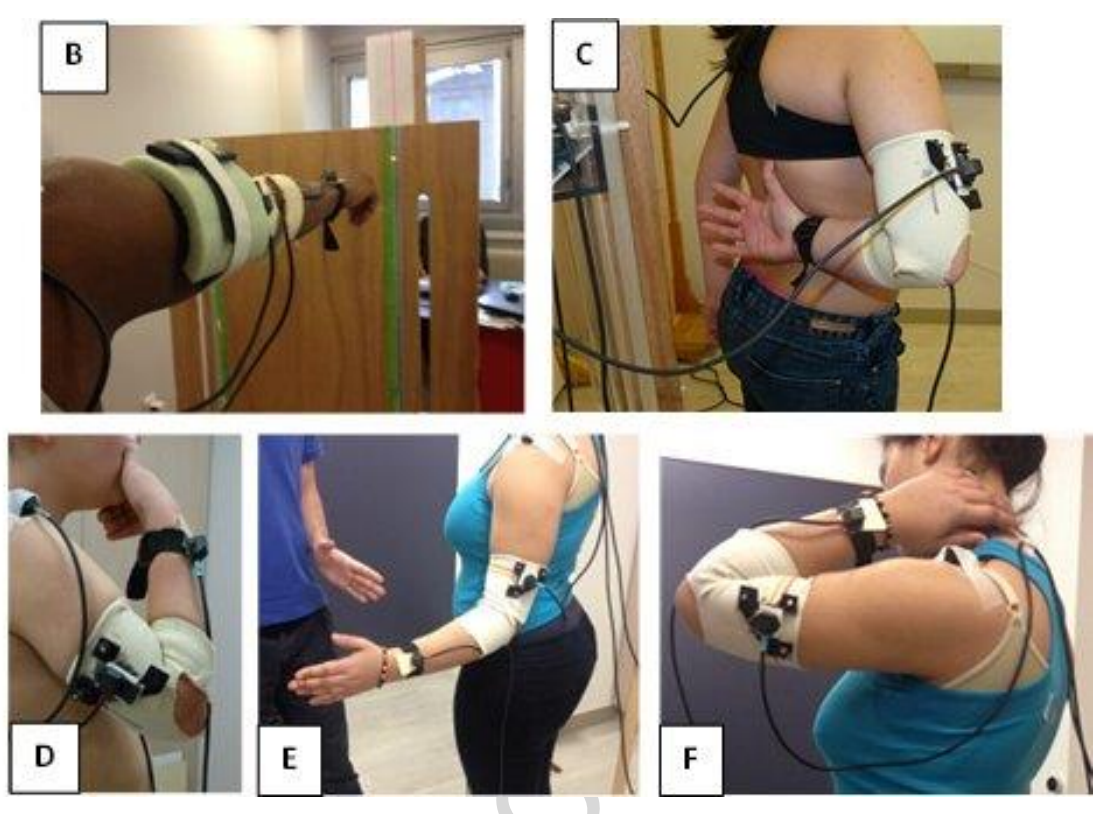

$(2)$ 

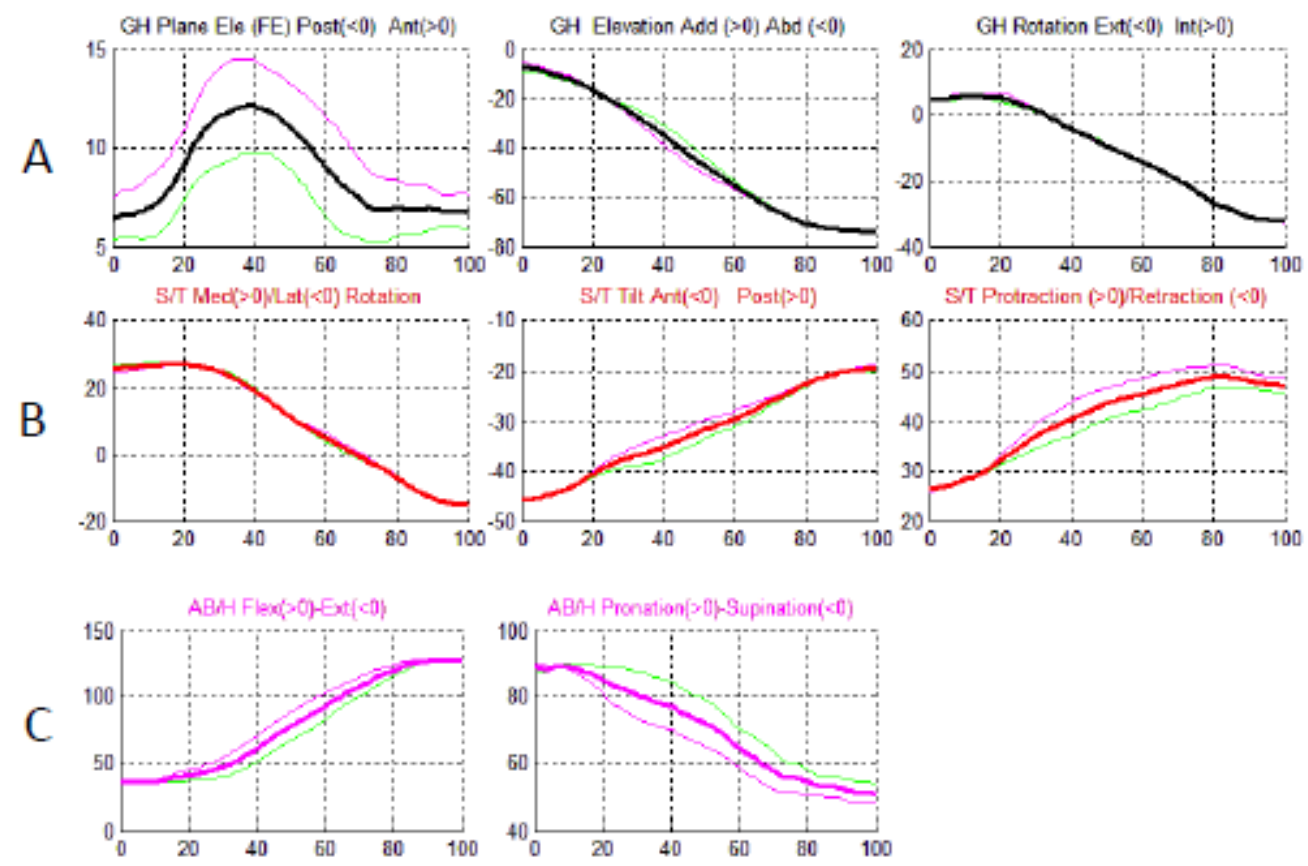

Fig. 2 


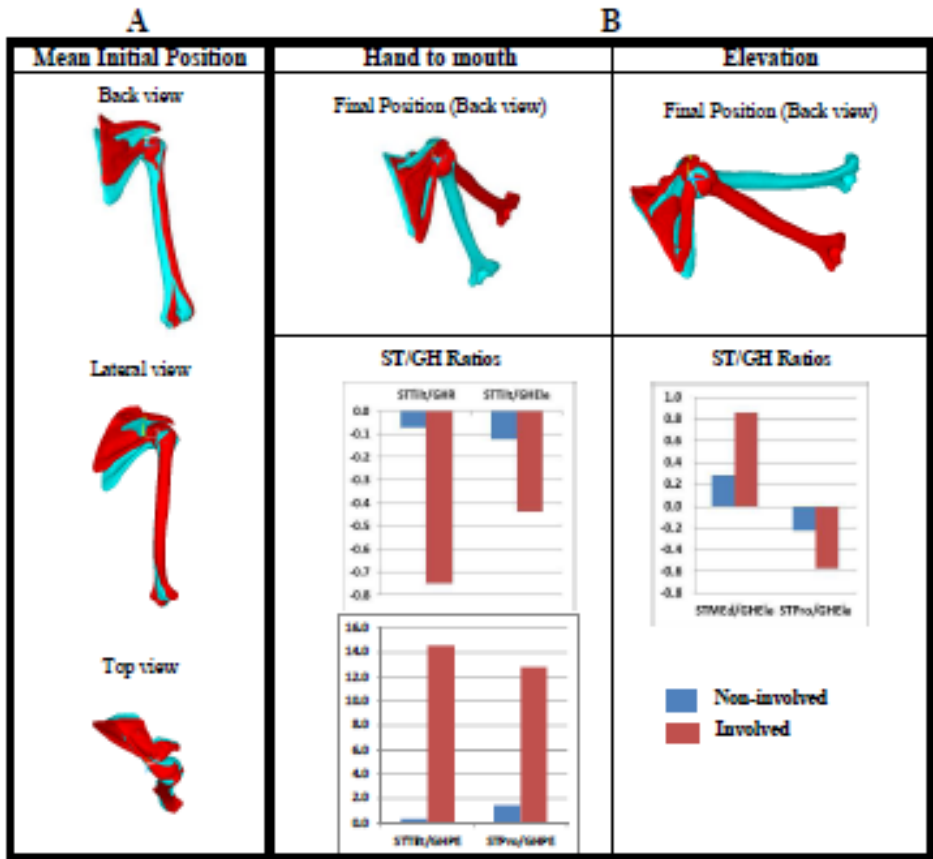

Fig. 3 

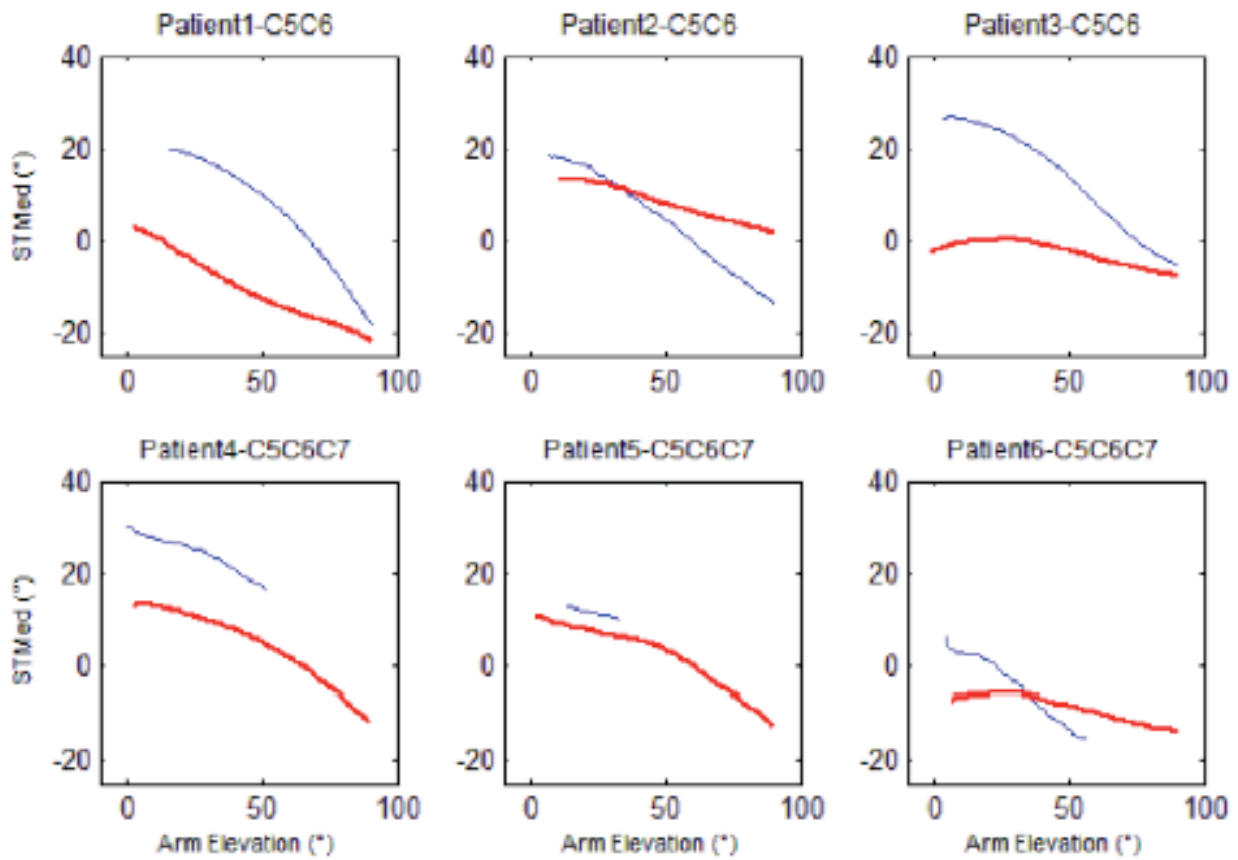

Fig. 4 


\section{Highlights}

- Kinematic analysis may be a valuable adjunct to clinical tools to assess shoulder motion dysfunction of obstetrical brachial plexus palsy patients.

- Scapulothoracic protraction and posterior tilt were significantly increased in the involved limb during the tasks Hand to mouth.

- Glenohumeral elevation excursion was significantly decreased in the involved limb during the hand to neck and the elevation tasks.

- The Scapulothoracic Protraction/Glenohumeral Elevation ratio was significantly increased in the involved limb during the hand to neck and the elevation tasks. 